\title{
Overexpression of monocarboxylate transporter 4 promotes the migration and invasion of non-carcinogenic L929 fibroblast cells
}

\author{
XIANGRU LI ${ }^{1}$, XIAOJU ZHOU ${ }^{2}$, YING LIU ${ }^{2}$, JINGJING FAN $^{2}$, HONGJING HUO ${ }^{2}$, \\ JINGJING YAO $^{2}$, LIN WANG $^{1}$ and NINGNING MA ${ }^{2}$ \\ ${ }^{1}$ School of Life Science and Biopharmaceutics; ${ }^{2}$ Wuya College of Innovation, \\ Shenyang Pharmaceutical University, Shenyang, Liaoning 110016, P.R. China
}

Received November 12, 2019; Accepted July 30, 2020

DOI: $10.3892 / \mathrm{ol} .2020 .12305$

\begin{abstract}
Metastasis is a primary contributor to the low survival rates of patients with cancer. Enhanced migration and invasion are two key features of the metastatic transformation of cancer cells. Furthermore, despite the fact that overexpression of the monocarboxylate transporter (MCT)1 and 4 proteins has been found to promote the migration or invasion of cancer cells, previous findings have not been conclusive and have even been contradictory. The majority of these previous studies have relied on the silencing or inhibition of MCT1/4 expression or function in highly metastatic cell lines. Silencing can be transient or incomplete, and inhibition can result in off-target effects. Employing a different approach, the present study stably transfected human MCT1 and MCT4 into the non-carcinogenic murine NCTC clone 929 (L929) cell line, which had undetectable endogenous MCT1 and MCT4 expression. It was observed that overexpression of MCT4, and not MCT1, promoted the migration and invasion of L929 cells. It was also found that overexpression of an inactive form of the MCT4 transporter with a single amino acid mutation failed to promote either migration or invasion, which suggested that MCT4 activity is required. Since an epidermal growth factor receptor (EGFR) inhibitor could reverse the effect of MCT4-overexpression, it was concluded that
\end{abstract}

Correspondence to: Professor Ningning Ma, Wuya College of Innovation, Shenyang Pharmaceutical University, 103 Wenhua Road, Shenyang, Liaoning 110016, P.R. China

E-mail: maningning@syphu.edu.cn

Abbreviations: MCT, monocarboxylate transporter; EGFP, enhanced green fluorescent protein; MMP, metalloproteinase; EGF, epidermal growth factor; EGFR, epidermal growth factor receptor; HGF, hepatocyte growth factor; c-Met, cellular mesenchymal to epithelial transition factor; FBS, fetal bovine serum; FACS, fluorescence-activated cell sorting

Key words: monocarboxylate transporters, migration, invasion, L929 cells
MCT4-overexpression exert its functions through modulating the EGF/EGFR pathway.

\section{Introduction}

Metastasis is an important driver of the high mortality rate of patients with cancer (1). Research on the impact of abnormal tumor cell metabolism on metastasis has gained attention in recent years. Most cancer cells rely on aerobic glycolysis for energy generation, which features high glucose uptake and high lactate production; a phenomenon widely known as the Warburg effect (2). To avoid the accumulation of large amounts of toxic lactic acid, cancer cells necessitate mechanisms that actively transport lactate out of cells. Two transporters of the monocarboxylate transporter (MCT) family, MCT1 and MCT4, have been shown to be associated with lactate transport (3). Their localization on the plasma membrane depends on the chaperone protein Basigin (CD147) (4).

Studies have shown that MCT1 and/or MCT4 are highly expressed in various types of cancer tissue, such as breast, lung and colorectal cancer tissues, and may serve as targets for cancer intervention (5-10). Moreover, when tumors progress to a higher grade or metastasis, a higher expression of MCT1 or MCT4 is typically observed (11-13), which suggests that their expression is associated with the migration or invasion of cancer cells. It has also been suggested that excess lactic acid that is secreted by cancer cells acidifies the tumor microenvironment and serves as a signal to promote the invasion and migration of cancer cells (14).

AZD3965, which is a specific inhibitor of MCT1 and MCT2, has been reported to induce the accumulation of intracellular lactate, which leads to the death of small cell lung cancer cells (15). Non-specific inhibitors of MCT transporters, including AR-C155858 and DIDS, can also block the function of MCT1 or MCT4 and lead to the death of breast and lung cancer cells, respectively $(16,17)$. Knocking down the expression of MCT4 was also been reported to inhibit the proliferation of gastric cancer cells (18).

In previous years, it has been reported that the expression of MCT in cancer cells is related to cell migration or invasiveness, but the results have been inconsistent among different studies. Studies have shown that knocking down the expression or inhibiting the function of MCT1 or MCT4 decreases 
the migration and invasion of breast cancer cells and nasopharyngeal carcinoma cells $(19,20)$. However, other studies have reported that the knockdown of MCT1 or MCT4 expression is only associated with invasion, but not migration $(17,21)$.

Silencing or knocking down MCT expression is difficult to standardize, since transfection is typically incomplete and silencing is transient $(22,23)$. Moreover, the transfection procedure itself is harmful to the cells due to the cytotoxicity of the transfection reagents (24). In this study, human MCT1 or MCT4 was overexpressed via stable transfection in L929 cells to evaluate their impact on cellular migration and invasion, which are two major traits of metastasis (25).

Metastatic cancer cells are commonly characterized by the enhancement of metalloproteinase (MMP) activity (26). Migration and invasion are regulated by the classical hepatocyte growth factor (HGF)/c-Met or epidermal growth factor (EGF)/EGF receptor (R)-mediated signal transduction pathways $(27,28)$. In the present study, the effects of MCT1 or MCT4 high expression on the migratory and invasive abilities of non-tumorigenic L929 cells were investigated. Preliminary investigations into the molecular mechanisms of MCT4 overexpression on migration and invasion were also performed.

\section{Materials and methods}

Plasmids preparation. The coding regions of human MCT1, MCT4, MCT4-R278Q or enhanced green fluorescent protein (EGFP) were synthesized with EcoRI and HindIII restriction sites (Genewiz, Inc.). These gene fragments and pcDNA3.0 vectors (Beijing Dingguo Changsheng Biotechnology Co., Ltd.) were treated with EcoRI and HindIII restriction endonuclease (Takara Bio, Inc.) at $37^{\circ} \mathrm{C}$ for $4 \mathrm{~h}$ and connected using DNA ligase (Takara Bio, Inc.) at $16^{\circ} \mathrm{C}$ for $12 \mathrm{~h}$. The respective gene sequence post-recombination was confirmed by Genewiz, Inc.

Transfection and generation of stable monoclonal cells. L929 [CCL-1 ${ }^{\mathrm{TM}}$; American Type Culture Collection (ATCC)], is a non-carcinogenic murine cell line and was cultured in Dulbecco's modified Eagle's medium (DMEM) (Gibco; Thermo Fisher Scientific, Inc.) supplemented with 10\% fetal bovine serum (FBS) (Sigma-Aldrich; Merck KGaA) in T-flasks. EGFP-L929, MCT1-L929, MCT4-L929 and MCT4-R278Q-L929 cells were established by transfecting respective plasmids into host L929 cells via electroporation. Electroporation was conducted at room temperature in $0.4 \mathrm{~cm}$ gapped electroporation cuvettes (aluminum electrode; $1 \mathrm{x} 1 \mathrm{~cm}$ ) using an electroporator (Gene Pulser XcellTM; Bio-Rad Laboratories, Inc.) according to manufacturer's instructions. Briefly, $1 \mu \mathrm{g} / \mu \mathrm{l}$ plasmids and $1.0 \times 10^{7}$ L929 cells were mixed in the electroporation cuvette at a total volume of $800 \mu 1$. Cell medium used for transfection was CSC03 medium with an osmolarity of 280-320 mOsm/kg (Vbiosci Co., Ltd.). Electroporation condition used an exponential protocol and was set at $900 \mu \mathrm{F}$ and $300 \mathrm{~V}$. After electroporation, the entire content in the electroporation cuvette was added into $200 \mathrm{ml}$ of pre-warmed CSC03 medium with $10 \%$ FBS and mixed well. A total of $100 \mu \mathrm{l}$ of the cell suspension was then added into each well of 96-well plates (Corning, Inc.), which corresponds to $\sim 5,000$ cells/well. Plates were then incubated at $37^{\circ} \mathrm{C}$ for $24 \mathrm{~h}$. An additional $100 \mu \mathrm{l}$ of CSC03 medium with $10 \%$ FBS

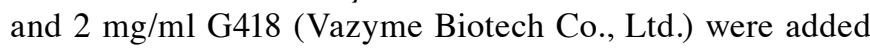
into each well. Plates are then returned to the incubator for 2 to 3 weeks. Cells that formed a single colony in the plate wells were scaled up into 24- and 6-well plates (Corning, Inc.) in DMEM with 10\% FBS. Clones were sub-cultured in 6-well plates with 1:5 split ratio every 3-4 days. Clones were screened for EGFP or MCT expression using flow cytometric analysis and confirmed by immunoblotting and immunofluorescence assays. Clones of interest were sub-cultured in T25 or T65 flasks (Corning, Inc.) in DMEM with 10\% FBS. Three high-expressing clones for each transfected L929 cells were used for the subsequent experiments.

Fluorescence-activated cell sorting (FACS). To screen for MCT expression, cells were detached from 6-well plates with trypsin (Sigma-Aldrich; Merck KGaA) and permeabilized with $0.1 \%$ Triton-X-100, as the primary antibody targeted the intracellular portions of the MCTs. Cells were incubated for $2 \mathrm{~h}$ at room temperature with primary antibodies against MCT1 (1:1,000; cat. no. ab85021; Abcam) or MCT4 (1:1,000; 22787-1-AP; ProteinTech Group, Inc.), and then with secondary fluorescein-conjugated goat anti-mouse IgG (1:5,000; cat. no. A-11001; Thermo Fisher Scientific, Inc.). Labeled cells were analyzed with a FACSCalibur flow cytometer (BD Biosciences) and Flowjo v7.6.1 program (FlowJo LLC). Importantly, EGFP-expressing cells were screened without labeling.

Western blot analysis. Proteins from whole cell lysates of L929, CHO, MCF7, HEK293, H526, A549, HeLa, U-87MG, and DMS114 (all purchased from ATCC; Fig. S1) were obtained using RIPA lysis buffer (Beijing Solarbio Science \& Technology Co., Ltd.) with a protease inhibitor cocktail (Sigma-Aldrich; Merck KGaA). Proteins at $10 \mu \mathrm{g} /$ lane were loaded onto a $12 \%$ acrylamide gel, resolved using SDS-PAGE; and were then transferred to a polyvinylidene difluoride membrane (Merck KGaA). After blocking with $5 \%$ bovine serum albumin (Sigma-Aldrich; Merck KGaA) for $2 \mathrm{~h}$ at room temperature, primary antibodies against MCT1, MCT4, CD147 (1:1,000; cat. no. ab119020; Abcam), or matrix metalloproteinase (MMP)2 (1:2,000; cat. no. 66366-1-Ig; ProteinTech Group, Inc.) were incubated overnight at $4^{\circ} \mathrm{C}$ and then washed with PBS-Tween20 (0.05\% Tween-20) and incubated with the secondary horseradish peroxidase (HRP)-conjugated goat anti-mouse IgG (1:5,000; cat. no. A-31430; Thermo Fisher Scientific, Inc.) at room temperature for $1 \mathrm{~h}$. Enhanced chemiluminescence (ECL) reagent (Vazyme Biotech Co., Ltd.) was used to detect the proteins, and the immunoblots were developed using the Image Scanner ChemiDoc MP (Bio-Rad Laboratories, Inc.) and analyzed by ImageJ v1.5.1 (National Institutes of Health).

Reverse transcription-PCR. Total RNA from whole cell lysates of L929, CHO, MCF7, HEK293, H526, A549, HeLa, U-87MG, and DMS114 (all purchased from ATCC; Fig. S1) was extracted using RNA purification Kit (CWBIO Co., Ltd.) according to the manufacturer's protocols. RNA concentration and purity were determined with a Nanodrop 2000 Analyzer 
(Thermo Fisher Scientific, Inc.). Purified RNA was reverse transcribed using the HiFiScript cDNA Synthesis Kit (CWBio). PCR was performed using the following thermocycling conditions: $94^{\circ} \mathrm{C}$ for $30 \mathrm{sec}, 55^{\circ} \mathrm{C}$ for $30 \mathrm{sec}$ and $72^{\circ} \mathrm{C}$ for $30 \mathrm{sec}$ on a PCR instrument (Mastercycler nexus, Eppendorf) using Super Multiplex PCR Mix (CWBio) according to the manufacturer's protocol. The primers for each gene were shown in Fig. S1C.

Immunofluorescence. MCT-transfected L929 cells were fixed in $4 \%$ paraformaldehyde for $30 \mathrm{~min}$ and permeabilized with $0.1 \%$ Triton-X-100 for 15 min. Cells were incubated with the aforementioned primary antibodies against MCT1, MCT4 or CD147 for $2 \mathrm{~h}$ and then treated with secondary fluorescein-conjugated antibodies for $30 \mathrm{~min}$. Nuclei were stained with DAPI (1:20; Thermo Fisher Scientific, Inc.) for $15 \mathrm{~min}$. The expression levels of MCT1, MCT4, or CD147 were observed under a fluorescence microscope (x20 magnification; DMI3000 B; Leica Microsystems $\mathrm{GmbH}$ ) and images were captured. All operations were performed at room temperature.

Lactate transport assay. MCT or EGFP transfected L929 cells were seeded into 96-well plates at a density of $5.0 \times 10^{4}$ cells/well. A final concentration of $1,5,10,30,50$, 100, 300 and $500 \mathrm{nM}$ AZD3965 in DMSO or pure DMSO (control) was added $24 \mathrm{~h}$ later, and the culture supernatant was sampled $72 \mathrm{~h}$ later. Lactate concentration was measured using a biosensor analyzer (Shandong Baisheng Biotechnology Co., Ltd.). Relative lactate concentrations were calculated by the ratio of lactate concentration between AZD3965-treated cells and pure DMSO treated samples (controls). The experiments were performed in triplicate.

Cell counting assay. L929 cells were seeded into 96-well plates at a density of $5.0 \times 10^{4}$ cells/well and cultured for 3 days. Cell proliferation was measured at 24,48 and $72 \mathrm{~h}$ using a Cell Counting Kit-8 (Gen-view Scientific, Inc.) according to the manufacturer's protocol. Relative cell proliferation was calculated based on the proliferation of EGFP-L929 cells at $24 \mathrm{~h}$. The experiments were performed in triplicate.

Wound healing assay for migration. MCT or EGFP transfected L929 cells were seeded into 24-well plates at a density of $1.0 \times 10^{6}$ cells/well and cultured until $\sim 90 \%$ confluency. A scratch was made using a sterile $200-\mu 1$ pipette tip, and detached cells were washed off using DMEM and replaced with new DMEM. For the EGFR inhibitor OSI-744 or c-Met inhibitor INCB28060 (both Selleck Chemicals), the medium was supplemented with $10 \mu \mathrm{M}$ OSI-744 or $5 \mu \mathrm{M}$ INCB28060 dissolved in DMSO, while the same volume of DMSO without inhibitors was used as the control. Wound healing was observed $48 \mathrm{~h}$ later under a phase contrast microscope (x4 magnification; Leica Microsystems $\mathrm{GmbH}$ ) and the cell migration rate was calculated by the percentage of wound closure. The EGFP transfected cells were used as negative controls for the MCT transfected cells. The experiments were performed in triplicate.

Transwell assays for migration and invasion. MCT or EGFP transfected L929 cells in serum-free medium were seeded at $1.0 \times 10^{5}$ cells/well in the inserts of 24 Transwell plates.
For the migration analysis, these cells were seeded directly into the inserts; meanwhile, for the invasion assay, $50 \mu \mathrm{l}$ of a 1:9 mixture of Matrigel (BD Biosciences) and culture medium were added to each upper compartment of the inserts and cured at $37^{\circ} \mathrm{C}$ for $24 \mathrm{~h}$ prior to cell seeding. Then, $10 \%$ FBS-containing medium was added to the bottom chamber of the Transwell plates. Cells on the lower surface of the insert were fixed in $4 \%$ paraformaldehyde at room temperature for $30 \mathrm{~min}$, stained with $0.1 \%$ crystal violet at room temperature for $30 \mathrm{~min}$ and examined under a microscope after $24 \mathrm{~h}$ (for migration) or $36 \mathrm{~h}$ of incubation (for invasion) at $37^{\circ} \mathrm{C}$. For the inhibition studies, either $10 \mu \mathrm{M}$ OSI-744 or $5 \mu \mathrm{M}$ INCB28060 was added to the cell culture medium prior to seeding the cells into inserts, while the same volume of DMSO without inhibitors was used as the control. The average number of migrated or invading cells from five designated optical fields under a phase contrast microscope (x40 magnification) was counted and averaged. The EGFP transfected cells were used as negative controls for the MCT transfected cells. The experiments were performed in triplicate.

Gelatin zymography. L929 cells were seeded into 6-well plates at a density of $5.0 \times 10^{6}$ cells/well and then incubated with serum-free medium at $37^{\circ} \mathrm{C}$ for $24 \mathrm{~h}$. Culture supernatants were collected and electrophoresed using 10\% SDS-PAGE containing 2\% gelatin (Corning, Inc.). After washing, the gel was cultured with a buffer that contains $5 \mathrm{mM} \mathrm{CaCl}_{2}$ and $1 \mu \mathrm{M} \mathrm{ZnCl}_{2}$ at $37^{\circ} \mathrm{C}$ for $48 \mathrm{~h}$. Afterwards, the gel was stained with Coomassie Blue R250 at room temperature for $3 \mathrm{~h}$ and decolorized with a solution that contained $5 \%$ methanol and $10 \%$ acetic acid. The band was scanned using Image Scanner (ChemiDoc MP; Bio-Rad, Laboratories, Inc.) and analyzed using ImageJ v1.5.1 (National Institutes of Health).

Statistical analysis. All statistical analysis was performed using GraphPad Prism 7.0 (GraphPad Software, Inc.). Two-tailed Welch's t-tests were used to compare results in two groups. One-way ANOVA or two-way ANOVA with Tukey's post hoc test was used to compare results in more than two groups. $\mathrm{P}<0.05$ was considered to indicate a statistically significant difference. The $\mathrm{P}$-values were calculated from the results of three independent experiments.

\section{Results}

Expression of EGFP, MCT1 or MCT4 in L929 cells. L929 is a non-carcinogenic murine cell line with undetectable murine MCT1 or MCT4 expression, analyzed using western blotting and PCR (Fig. S1A and B). The impact of overexpression of human MCT1 or MCT4 in L929 cells on cellular migration and invasion was investigated. To avoid using a pool of cells with both transfected and non-transfected cells, transfected cells were cloned to select only high-expressing clones. To overcome clone-to-clone variations, a panel of three stable clones was used for each genetic construct. The negative controls were three clones of L929 cells with high expression levels of the mock EGFP.

For transfection, pcDNA3.0-EGFP, pcDNA3.0-MCT1 and pcDNA3.0-MCT4 plasmids were constructed and verified (Fig. S2A-C). The plasmids were then used to transfect L929 

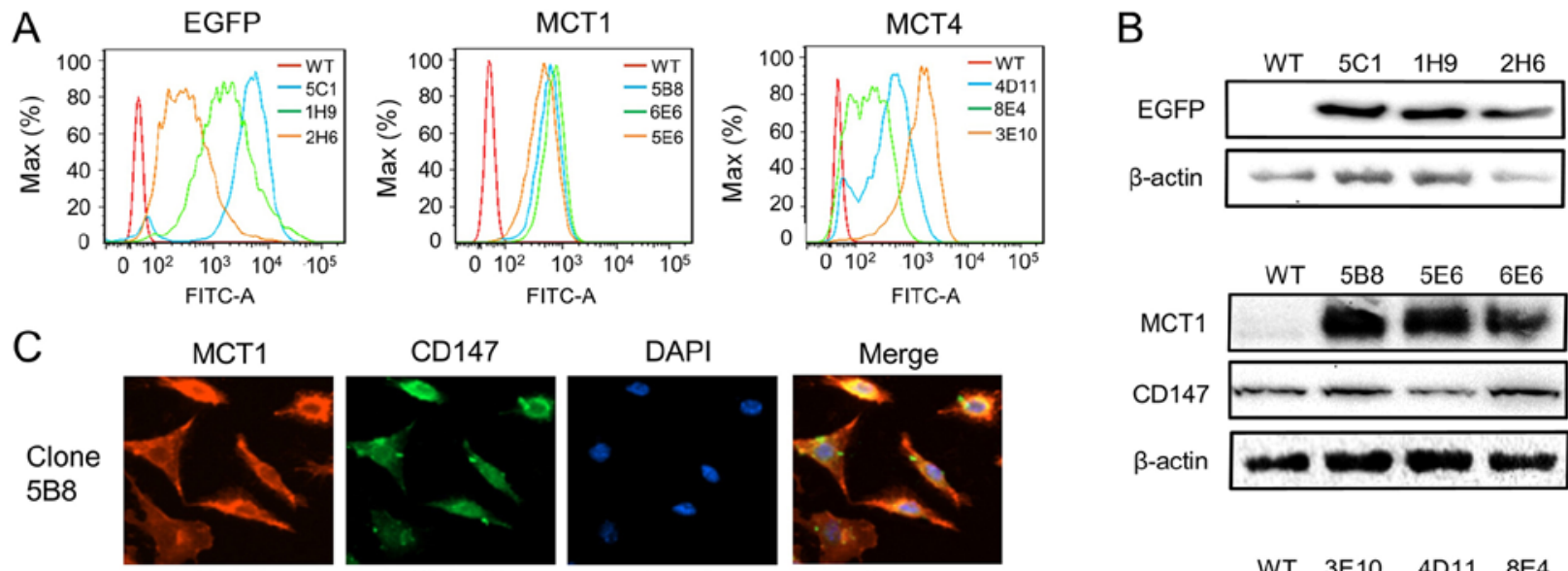

DAPI
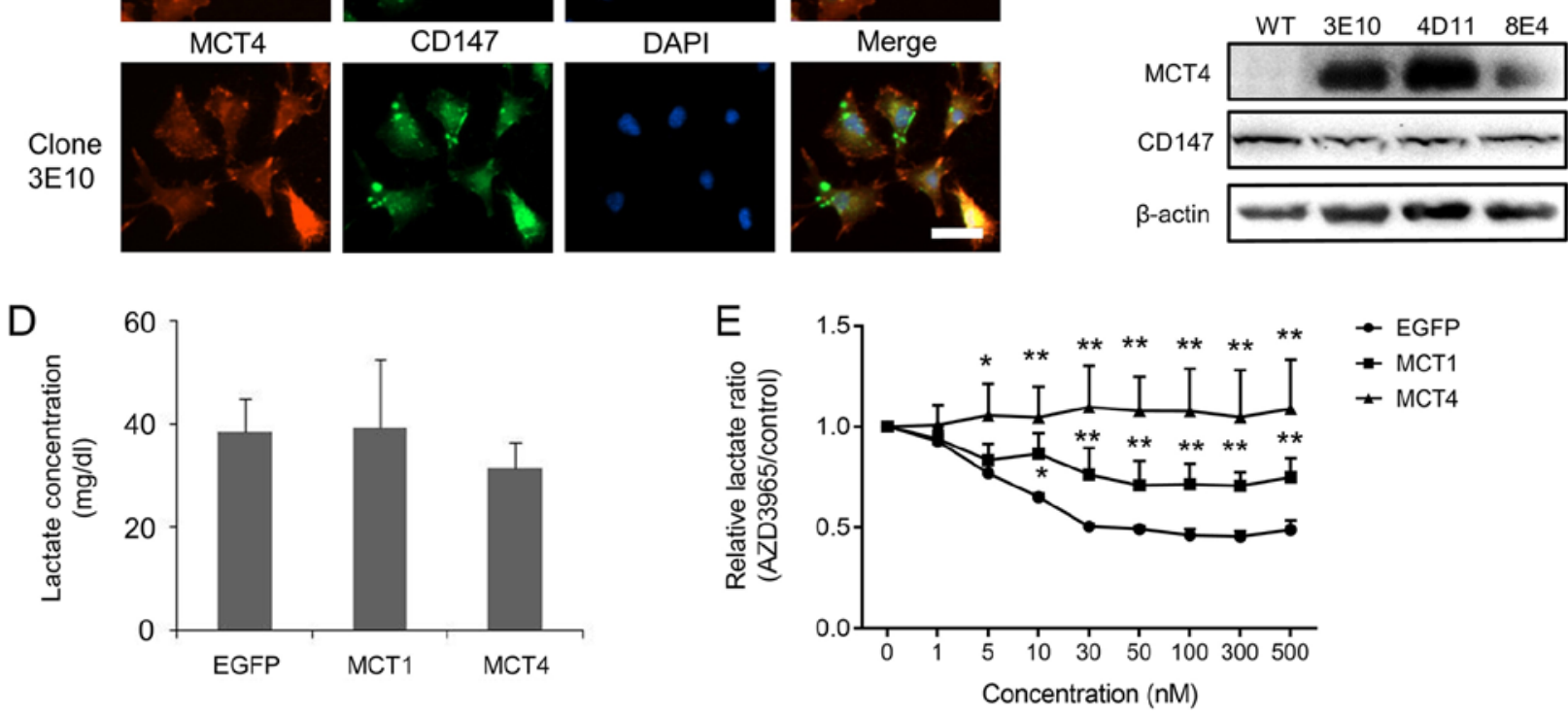

Figure 1. Overexpression of EGFP, MCT1 and MCT4 in L929 cells. (A) Overexpression of EGFP, MCT1 and MCT4 was confirmed using flow cytometry for EGFP-L929, MCT1-L929 and MCT4-L929 clones. (B) Overexpression of EGFP, MCT1, and MCT4, as well as expression of endogenous CD147 were confirmed using western blotting. (C) Immunofluorescence was performed to investigate the expression of transfected MCT1 or MCT4. Co-expression of MCT and CD147 was observed in clone 5B8 of MCT1-L929 and clone 3E10 of MCT4-L929 cells. Scale bar, $30 \mu \mathrm{m}$. (D) Lactate concentration in the culture medium of MCT1-, MCT4- and EGFP-transfected cells. No significant differences were observed among different panel of clones. Data were analyzed using a two-tailed Welch's t-test. (E) Normalized lactate concentration in the culture medium of AZD3965-treated cells to respective control cells that received only AZD3965 solvent (DMSO). The MCT1/2 inhibitor, AZD3965, reduced lactate secretion of EGFP-L929 cells in a dose-dependent manner, but MCT1-L929 and MCT4-L929 cells were partially or completely resistant to AZD3965. The results in (D) and (E) were the average of three independent experiments using three different clones, and each error bar indicates one standard deviation. P-values shown in (E) were calculated using one-way ANOVA with a Tukey's post hoc test. ${ }^{*} \mathrm{P}<0.05,{ }^{* *} \mathrm{P}<0.01$ vs. EGFP. EGFP, enhanced green fluorescent protein; MCT, monocarboxylate transporter.

cells and transfected cells were single-cell cloned. Clones were ranked by FACS (Fig. S2D-F) and western blotting (Fig. S2G-I) for MCT1, MCT4 or EGFP expression. Three high MCT1 expressing clones (MCT1-L929 5B8,5E6 and 6E6), three high MCT4 expressing clones (MCT4-L929 3E10, 4D11 and 8E4), and three EGFP expressing clones (EGFP-L929 1H9, 2H6 and $5 \mathrm{C} 1)$ were chosen for subsequent investigations. Throughout the investigation, the expression of MCT1, MCT4 or EGFP in the nine chosen clones were monitored concurrently. As shown in Fig. 1A and B, high expression of MCT1, MCT4 or EGFP was well maintained during the investigation. Human CD147, which is the molecular chaperone of MCT1 and MCT4 that assists in guiding MCT proteins to the membrane (4), was not co-transfected. However, mouse CD147 was present in all clones and showed similar distributions as those of MCT1 (5B8) and MCT4 (3E10) (Fig. 1C). Other clones of MCT1- and MCT4-L929 showed similar results. Overexpression of MCT1 or MCT4 did not increase lactate secretion of L929 cells
(Fig. 1D). Nevertheless, it rendered L929 cells more resistant to AZD3965, which is an inhibitor of MCT1 and MCT2 (Fig. 1E). At a concentration of $10 \mathrm{nM}$ AZD3965 or higher, both MCT1-L929 and MCT4-L929 cells had a significant reduction on lactate secretion compared with EGFP-L929 cells. The lactate secretion of EGFP-L929 cells was reduced by $~ 50 \%$; in contrast, the lactate secretion of MCT1-L929 cells was reduced by only $30 \%$ and that of MCT4-L929 cells was not reduced at all. These observations suggested that lactate metabolism and transportation of L929 cells are tightly regulated and may not be affected by overexpression of MCT1 or MCT4. However, once lactate transportation was disrupted, the overexpression of MCT1 or MCT4 partially or completely rescued the normal phenotype.

Migration and invasion of MCT1- or MCT4-transfected L929 cells. Migration and invasion are two key features of metastasis (25). Migration was analyzed using wound 
A

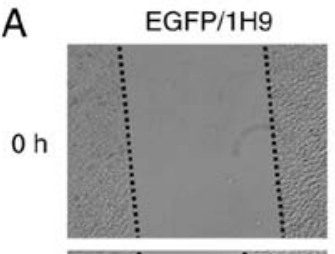

$48 \mathrm{~h}$

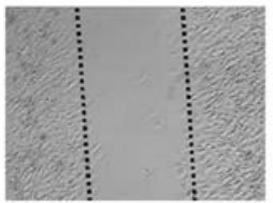

B

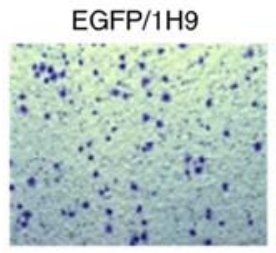

C

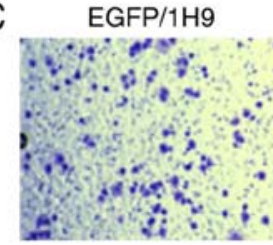

MCT1/5B8

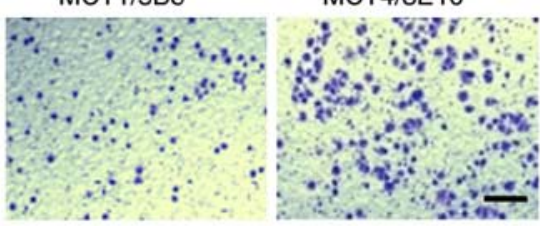

MCT $1 / 5 B 8$
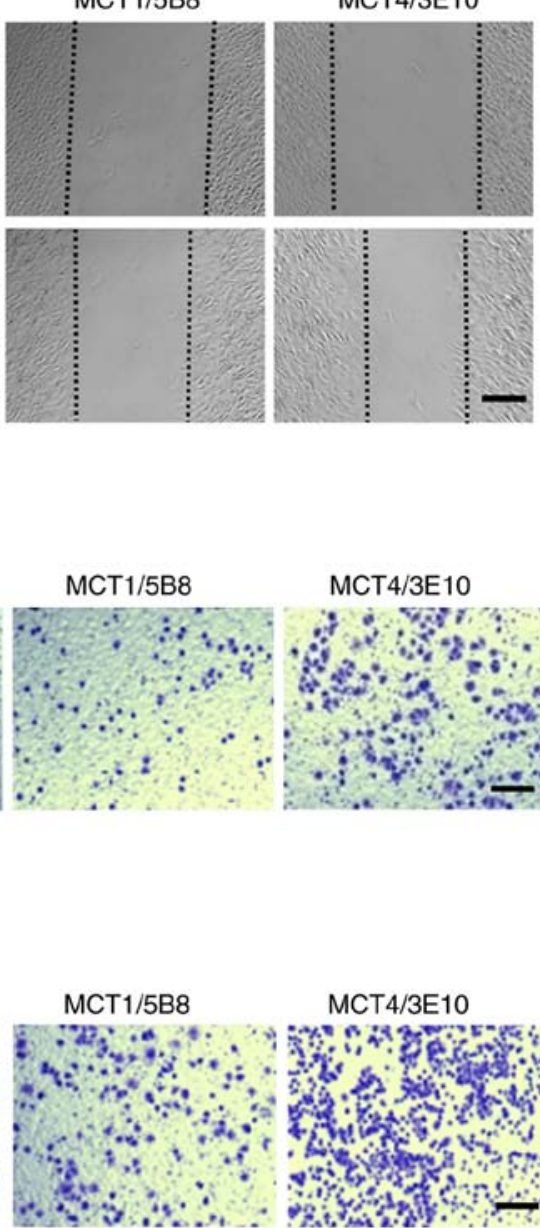

MCT4/3E 10

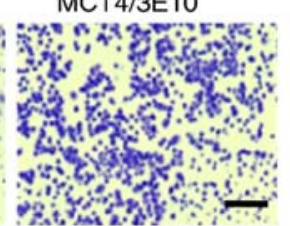

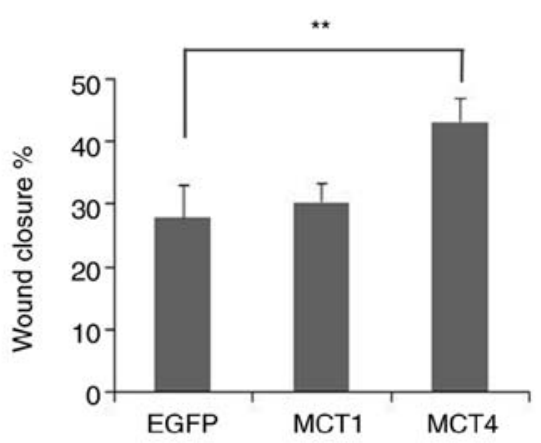
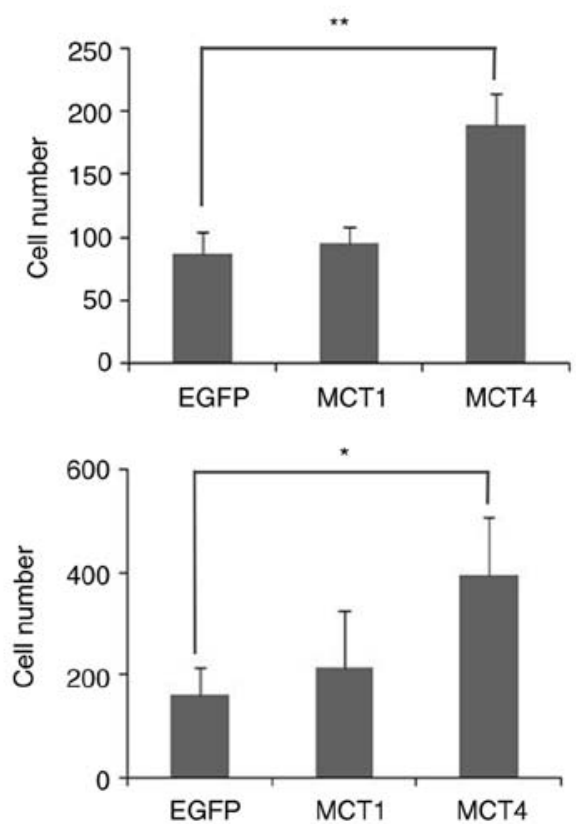

Figure 2. Migration and invasion of MCT1- and MCT4-transfected L929 cells. (A) Wound healing assay. Representative images of scratch closing of EGFP-L929 (1H9), MCT1-L929 (5B8) and MCT4-L929 (3E10) cells at 0 and 48 h post-scratch are shown. Scale bar, $300 \mu$ m. Percentage of wound closure, calculated as the average of three parallel clones, at $48 \mathrm{~h}$ post scratch is shown to the right. (B) Migration assay. Representative images of EGFP-L929 (1H9), MCT1-L929 (5B8) and MCT4-L929 (3E10) cells that migrated through a migration filter are shown after the cells were seeded on the top chambers for $36 \mathrm{~h}$. The average number of cells that adhere to the lower chamber is presented to the right. (C) Invasion assay. Cells were plated onto Matrigel invasion chambers for 36 h. Representative images of EGFP-L929 (1H9), MCT1-L929 (5B8) and MCT4-L929 (3E10) cells that invaded through the Matrigel invasion filters are shown. The average number of cells that adhere to the lower chamber is presented to the right. The average results in (A), (B) and (C) were from three independent experiments using three different clones, and each error bar represents one standard deviation. P-values shown in (A), (B) and (C) were calculated using one-way ANOVA with a Tukey's post hoc test. ${ }^{*} \mathrm{P}<0.05,{ }^{* *} \mathrm{P}<0.01$ vs. EGFP. EGFP, enhanced green fluorescent protein; MCT, monocarboxylate transporter.

healing and Transwell migration assays, while invasion was analyzed using a Transwell invasion assay. As shown in Fig. 2A, the migration rate of MCT4-L929 clones was significantly faster compared with that of the EGFP control clones $(\mathrm{P}<0.01)$, while the MCT1-L929 clones were not different from the control in terms of migration rate. A Transwell migration assay confirmed these results. As shown in Fig. 2B, more than twice as many cells passed through Transwell membranes for the MCT4-overexpression clones $(\mathrm{P}<0.01)$, while MCT1 overexpression clones behaved similar to the control clones. In addition, the invasiveness of L929 cells was increased for MCT4-expressing cells, and not MCT1-overexpressing cells ( $\mathrm{P}<0.05$; Fig. 2C). Approximately twice as many cells passed through the Matrigel base for MCT4-L929 cells compared with both control and MCT1-L929 cells. These results indicated that MCT4, but not MCT1, promoted both migration and invasion of L929 cells.
Migration and invasion of dysfunctional MCT4-transfected L929 cells. It was investigated whether the function of MCT4 is required for its promotion of migration and invasion of L929 cells. A single amino acid mutation, known as R278Q, causes MCT4 to be completely dysfunctional (29). A panel of L929 clones transfected with the MCT4-R278Q gene were screened using FACS and western blotting (Fig. S3A and B). Three MCT4-R278Q-L929 high-expressing clones (8E4R, 8D6 and 9G2) were selected, as shown in Fig. 3A-C. It was first confirmed that the MCT4-R278Q mutants lost their ability to engage in lactate transportation. As shown in Fig. 3D, the lactate secretion of MCT4-R278Q-L929 cells was reduced by AZD3965 by $\sim 50 \%$, which was similar to that of the negative control (EGFR-L929) cells, whereas AZD3965 treatment of MCT4-L929 cells showed no inhibitory effect. Fig. 3D indicated that MCT4-R278Q-L929 cells could not compensate for the inhibition of AZD3965, in contrast to the wild-type MCT4-L929 cells, and behaved similarly to the negative 
A

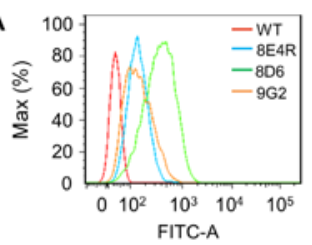

C

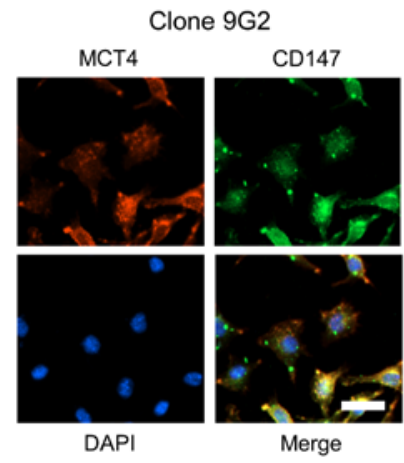

B

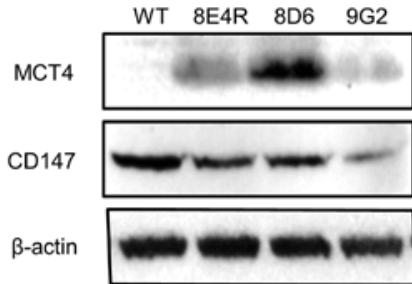

D

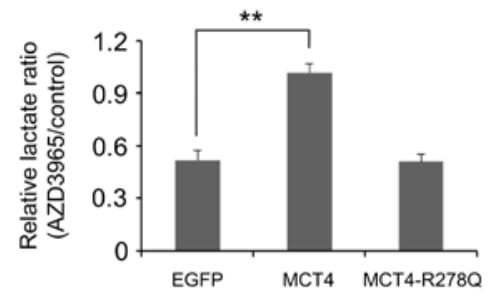

E
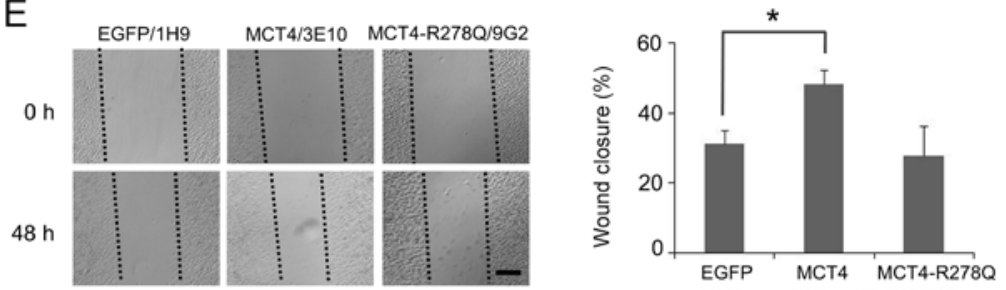

$\mathrm{F}$
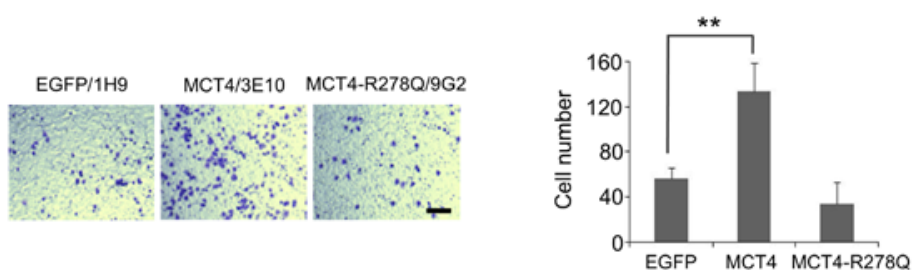

G

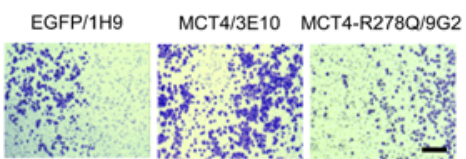

Figure 3. Mutated MCT4 without lactate transportation activity does not promote migration or invasion of L929 cells. A panel of three clones with high expression of MCT4-R278Q was obtained, as evidenced by (A) flow cytometry and a (B) western blotting. (C) Co-expression of MCT4-R278Q and CD147 was observed with clone 9G2. Scale bar, $30 \mu \mathrm{m}$. (D) Normalized concentration of lactate in the culture medium of AZD3965-treated cells compared with that of the corresponding cells that are treated with only the solvent. MCT4-R278Q-L929 cells lost the ability to compensate for the inhibition mediated by AZD3965 in L929 cells compared with MCT4-L929 cells. (E) Wound healing assay. MCT4-R278Q-L929 (9G2) cells showed a similar migration rate as EGFP-L929 (1H9) cells, which was much slower compared with that of wild-type MCT4-L929 (3E10) cells. Scale bar, $300 \mu \mathrm{m}$. The average percentage of wound closure at $48 \mathrm{~h}$ is shown to the right. (F) Representative images of EGFP-L929 (1H9), MCT4-L929 (3E10) and MCT4-R278Q-L929 (9G2) cells that crossed through migration filters $36 \mathrm{~h}$ post seeding are shown. The average number of cells that adhered to the lower chamber is shown to the right. (G) Representative images of EGFP-L929 (1H9), MCT4-L929 (3E10) and MCT4-R278Q-L929 (9G2) cells that invaded through the Matrigel-coated filters $36 \mathrm{~h}$ after seeding are shown. The average number of cells that adhered to the lower chamber is presented to the right. The average is from three independent experiments that use three different clones, and each error bar represents one standard deviation. P-values shown in (D), (E), (F), and (G) were calculated using one-way ANOVA with a Tukey's post hoc test. ${ }^{*} \mathrm{P}<0.05,{ }^{* *} \mathrm{P}<0.01$ vs. EGFP. EGFP, enhanced green fluorescent protein; MCT, monocarboxylate transporter.

control cells, indicating that the R278Q mutation completely annihilated the lactate transportation function of MCT4. The promotion of migration and invasion by MCT4 was lost with the R278Q mutation, as shown in Fig. 3E-G, where MCT4-R278Q cells behaved similarly to the negative control, EGFP-L929 cells, but not to the MCT4-L929 cells with active lactate transportation function. As the expression level of MCT4 and MCT4-R278Q on respective cells was similar, our observation suggests that cellular migration and invasion were associated with the transportation function of MCT4.

\section{The EGF/EGFRpathwayinmigrationandinvasion is promoted} by MCT4. EGF/EGFR- and HGF/c-Met-mediated signaling pathways are two classical pathways that are associated with the regulation of cell migration and invasion $(30,31)$. It was investigated whether these two pathways were involved in the 
A
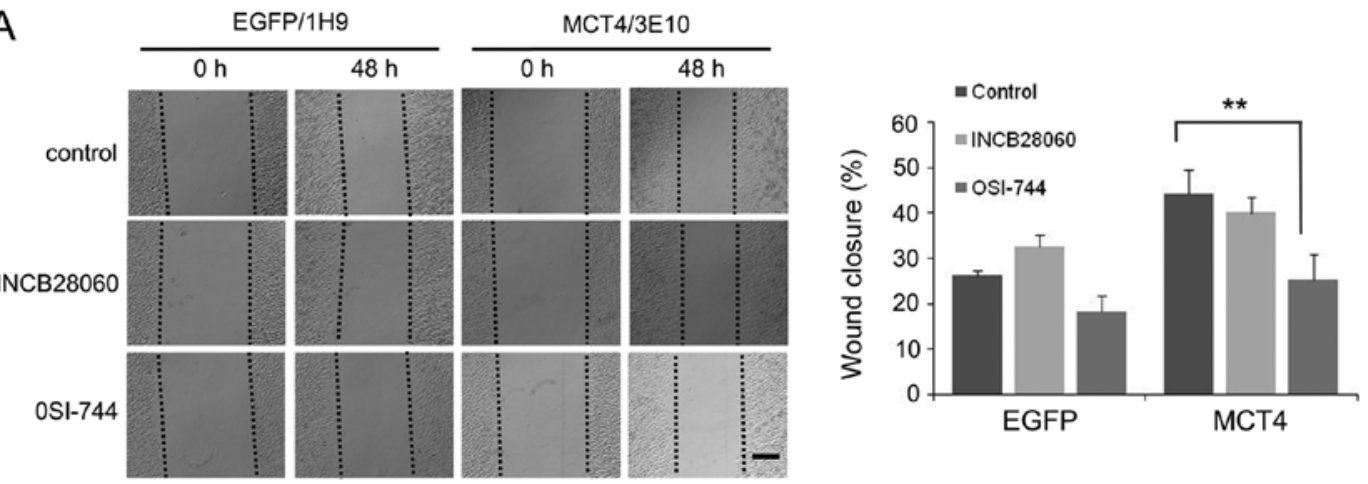

B
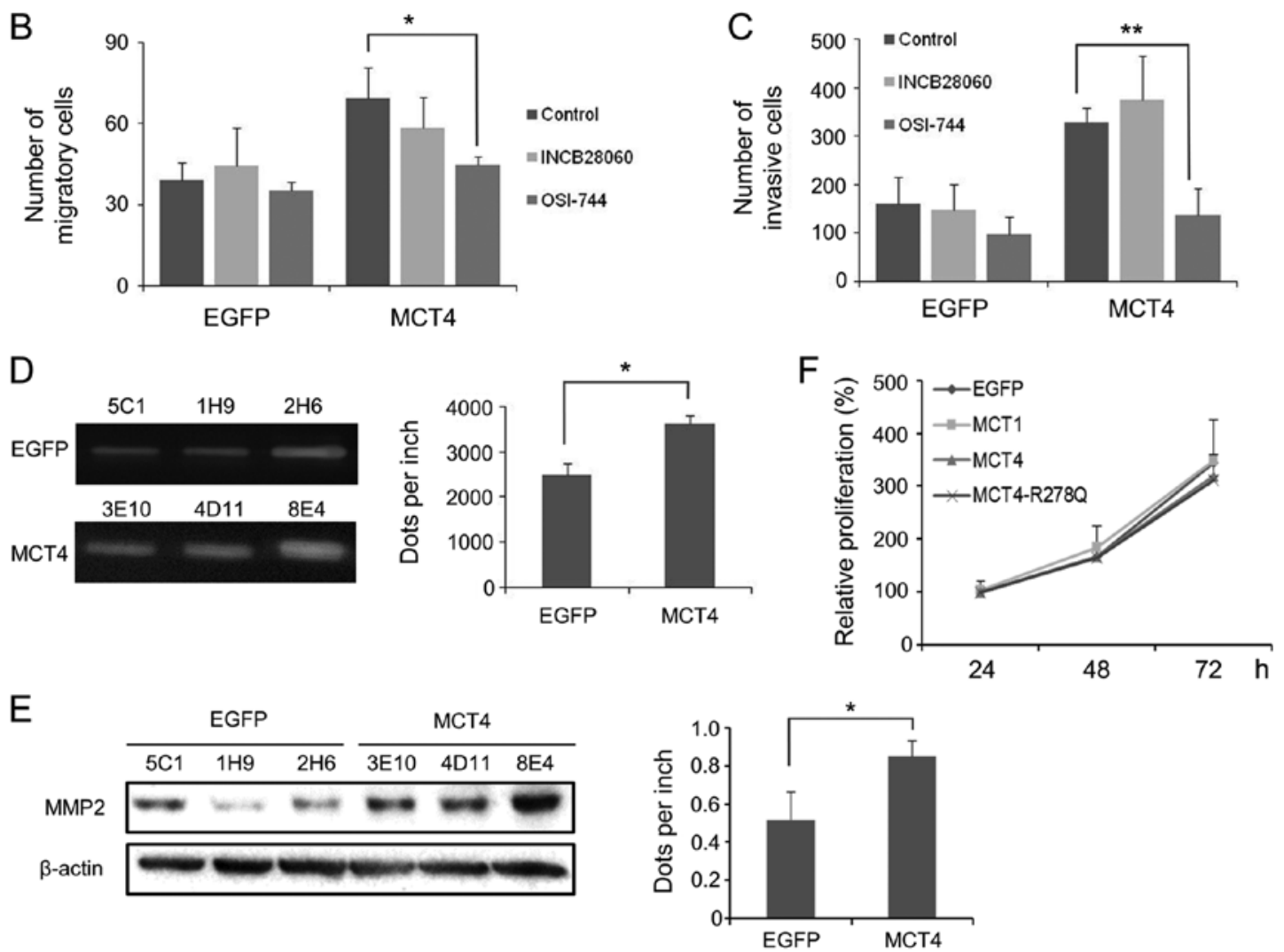

Figure 4. The EGF/EGFP-mediated signaling pathway may be associated with the enhanced migration and invasion of MCT4-L929 cells. (A) Representative wound healing images of EGFP-L929 (1H9) and MCT4-L929 (3E10) cells that were treated with the EGFP inhibitor OSI-744 or the c-Met inhibitor INCB28060 at 0 and $48 \mathrm{~h}$ post-scratching are shown. Scale bar, $300 \mu \mathrm{m}$. The average percentage of wound closure at $48 \mathrm{~h}$ is presented to the right. (B) EGFP inhibitor OSI-744- or the c-Met inhibitor INCB28060-treated MCT4-L929 cells were plated directly onto migration chambers for 36 h. The average numbers of cells that adhere to the lower chamber are presented. (C) EGFP inhibitor OSI-744- or c-Met inhibitor INCB28060-treated MCT4-L929 cells were plated onto Matrigel invasion chambers and cultured for $36 \mathrm{~h}$. The average number of cells that adhered to the lower chamber is presented. (D) MMP2 activity of the EGFP-L929 and MCT4-L929 cells was detected by a gelatin zymography assay. The gray value of the strip is presented to the right. (E) MMP2 levels were determined using western blotting. The gray value of the MMP2 bands is presented to the right. (F) The cell viability of EGFP-L929, MCT1-L929, MCT4-L929, and MCT4-R278Q-L929 cells during a 3-day culture. The average is of three independent experiments using three different clones, and each error bar represents one standard deviation. P-values in (A), (B) and (C) were calculated using two-way ANOVA with a Tukey's post hoc test, and P-values in (D) and (E) were calculated using two-tailed Welch's t-test. ${ }^{*} \mathrm{P}<0.05,{ }^{* *} \mathrm{P}<0.01$ vs. OSI-744 or EGFP. EGFP, enhanced green fluorescent protein; MCT, monocarboxylate transporter; MMP, matrix metalloproteinase.

promotion of migration and invasion of MCT4-L929 cells. Both MCT4-L929 cells and control EGFP-L929 cells were treated with either the EGFR inhibitor OSI-744 or the c-Met inhibitor INCB28060 to assess their impact on migration and invasion. Treatment with the EGFR inhibitor OSI-744 decreased the migration rate of MCT4-L929 cells, as demonstrated by both the wound healing and Transwell migration assays $(\mathrm{P}<0.01$ and $\mathrm{P}<0.05$; Fig. $4 \mathrm{~A}$ and $\mathrm{B}$, respectively). However, the c-Met inhibitor had no impact, as shown in Fig. 4A and 4B. Similar phenomena were observed in the invasion study. Fig. 4C shows that the invasiveness of MCT4-L929 cells was significantly decreased by OSI-744 ( $\mathrm{P}<0.01)$, but not INCB28060. These results indicated that the EGF/EGFR pathway may be involved in the metastatic transformation of L929 cells by MCT4.

As MMPs are commonly highly expressed with highly active in metastatic cells, presumably via the EGF/EGFR pathway (32), the activity and expression of MMPs in MCT4-L929 cells were investigated through gelatin zymog- 
raphy and western blotting. As shown in Fig. 4D, MMP2 activity in the culture medium of MCT4-L929 cells was increased compared with EGFP-L929 cells ( $\mathrm{P}<0.05)$. MMP2 levels in MCT4-L929 cells were also elevated ( $\mathrm{P}<0.05$; Fig. 4E), which is considered to be related to cell invasion. However, MMP9 expression was not detected in any L929 clone (data not shown). Cell proliferation rate of MCT4-L929 cells was similar to that of MCT1-, MCT4-R278Q-, and the negative control EGFP-L929 cells (Fig. 4F), hence the possibility of the higher migration and invasion rate of MCT4-L929 cells being a result of higher cellular proliferation rate could be ruled out.

\section{Discussion}

Transfected human MCT1 or MCT4 is active in murine L929 cells. Although overexpression of MCT1 and MCT4 does not alter lactate metabolism or transportation, such overexpression was found to counteract the effects of the MCT1/2 inhibitor AZD3965 (Fig. 1D and E). CD147, which is the chaperone protein that helps direct MCT transporters to the plasma membrane (4), was not co-transfected. Nevertheless, the function of endogenous murine CD147 seemed to be sufficient to direct recombinant MCT proteins to the plasma membrane and support their function.

It has been reported that MCF7 cells that are transfected with MCT1 show both higher tumorigenicity in nude mice and enhanced in vitro invasion (33). Since the present screening study demonstrated that MCF7 cells express MCT4, albeit at low levels, MCF7 cells were not used in the present study.

A panel of three high expression clones was used for each genetic construct. The negative control was a panel of three clones with high expression of EGFP, which was a mock protein that underwent the same clone selection process. Studies on these transgenic cell lines demonstrated that overexpression of human MCT4, but not human MCT1, promoted the migration and invasion of the noncarcinogenic L929 cell line. In addition, it was observed that the activity of MCT4 is required for migration and invasion promotion. When L929 cells were transfected with an inactive MCT4 mutant, neither migration nor invasion increased. Inactive MCT4 has a single amino acid mutation, known as R278Q, which was previously reported to be inactive (29). Moreover, Fig. 3D shows that the R278Q mutant lost its ability to counteract the function of AZD3965. This observation suggested that the MCT4 protein needs to be functional to promote cellular migration and invasion. No previous studies have evaluated the impact of inactivated MCT4 or MCT1 on cell migration and invasion, to the best of our knowledge. However, MCT1 has been suggested to promote metastasis of human SiHa cells independent of its activity (34), whereby a MCT1 inhibitor blocks lactate transportation, but does not reduce the migration or invasion rate of SiHa cells. It is unknown if the discrepancy on cell migration and invasion between the present findings and those of the study on $\mathrm{SiHa}$ cells is due to differences in cell lines, methods or differences between MCT1 and MCT4. It would be worthwhile to investigate whether other substrates, besides lactate, that are also transported by MCT4, but not by MCT1, contribute to enhanced migration and invasion.
To investigate possible mechanisms between overexpression of MCT4 and migration or invasion, two signaling pathways were examined: The HGF/c-Met and EGF/EGFR signaling pathways. The migration and invasion of MCT4-L929 cells were decreased to the same level as control cells by the EGFR inhibitor OSI-744, but not by the c-Met inhibitor INCB28060. Activated EGFR has been suggested to promote the invasiveness of MCF7 cells by upregulating MMP2 and MMP9 (35). Although MMP9 expression was not detected in L929 cells, it was demonstrated that MMP2 activity was slightly upregulated in the MCT4-L929 cells.

In conclusion, the present study reported the complex impact of the overexpression of MCT transporters on cell migration and invasion. The finding that the overexpression of MCT4 promotes both migration and invasion is consistent with those of previous studies, while the finding that the overexpression of MCT1 promotes migration and invasion is inconsistent. Second, it was demonstrated that, while MCT4 activity is critical for the migration and invasion of L929 cells, the activity of MCT1, which is similar to MCT4 in respect to lactate transportation function, had no impact on L929 migration and invasion. Finally, it was revealed that overexpression of active human MCT4 increased migration and invasion capability of non-carcinogenic L929 cells. Inhibition of MCT4 activity may serve as a potential therapeutic strategy to eliminate metastatic tumor cells. As such, the stably transfected MCT1- and MCT4-L929 cell lines may be used as valuable model cell lines both in vitro and in vivo to further investigate the mechanisms between overexpression of MCT1 and MCT4 and cell signaling, metabolism and tumorigenicity.

\section{Acknowledgements}

The authors would like to thank Dr Yu Yang and Ms. Xiaonan Ma (Shenyang Pharmaceutical University) for their technical support and instruction.

\section{Funding}

This work was funded by the Doctoral Start-up Foundation of Liaoning Province (grant no. 20170520316), the Junior Teacher Career Development Support Plan Foundation of Shenyang Pharmaceutical University (grant no. ZQN2016019), the Department of Education of Liaoning Province (grant no. 201610163L24), and Significant New Drug Development Project of Ministry of Science and Technology (grant no. 2019ZX09732-001) of China.

\section{Availability of data and materials}

The datasets used and/or analyzed during the present study are available from the corresponding author on reasonable request.

\section{Authors' contributions}

XL was a major contributor in the writing of the manuscript and was responsible for the statistical analysis. $\mathrm{XZ}$ and YL contributed to the development of all MCT and EGFP overexpressing L929 cell lines. JF and HH contributed to the migration and invasion experiment. JY contributed to 
the western blotting, fluorescence-activated cell sorting and immunofluorescence assays. XL and LW assisted with the design of the study. NM contributed to the conception and design of the study and revised the manuscript. All authors read and approved the final manuscript.

\section{Ethics approval and consent to participate}

Not applicable.

\section{Patient consent for publication}

Not applicable.

\section{Competing interests}

The authors declare that they have no competing interests.

\section{References}

1. Reuben JM, Krishnamurthy S, Woodward W and Cristofanilli M: The role of circulating tumor cells in breast cancer diagnosis and prediction of therapy response. Expert Opin Med Diagn 2: 339-348, 2008

2. Ngo H, Tortorella SM, Ververis K and Karagiannis TC: The Warburg effect: Molecular aspects and therapeutic possibilities. Mol Biol Rep 42: 825-834, 2015.

3. Halestrap AP: The monocarboxylate transporter family-Structure and functional characterization. IUBMB Life 64: 1-9, 2012.

4. Kirk P, Wilson MC, Heddle C, Brown MH, Barclay AN and Halestrap AP: CD147 is tightly associated with lactate transporters MCT1 and MCT4 and facilitates their cell surface expression. EMBO J 19: 3896-3904, 2000.

5. Li Z, Wu Q, Sun S, Wu J, Li J, Zhang Y, Wang C, Yuan J and Sun S: Monocarboxylate transporters in breast cancer and adipose tissue are novel biomarkers and potential therapeutic targets. Biochem Biophys Res Commun 501: 962-967, 2018.

6. Pinheiro C, Reis RM, Ricardo S, Longatto-Filho A, Schmitt F and Baltazar F: Expression of monocarboxylate transporters 1, 2, and 4 in human tumours and their association with CD147 and CD44. J Biomed Biotechnol 2010: 427694, 2010.

7. Jeon JY, Lee M, Whang SH, Kim JW, Cho A and Yun M: Regulation of acetate utilization by monocarboxylate transporter 1 (MCT1) in hepatocellular carcinoma (HCC). Oncol Res 26: 71-81, 2018.

8. Pinheiro C, Longatto-Filho A, Azevedo-Silva J, Casal M, Schmitt FC and Baltazar F: Role of monocarboxylate transporters in human cancers: State of the art. J Bioenerg Biomembr 44: 127-139, 2012.

9. Pinheiro C, Longatto-Filho A, Scapulatempo C, Ferreira L, Martins S, Pellerin L, Rodrigues M, Alves VA, Schmitt F and Baltazar F: Increased expression of monocarboxylate transporters 1, 2, and 4 in colorectal carcinomas. Virchows Arch 452: 139-146, 2008

10. Ruan Y, Zeng F, Cheng Z, Zhao X, Fu P and Chen H: High expression of monocarboxylate transporter 4 predicts poor prognosis in patients with lung adenocarcinoma. Oncol Lett 14: 5727-5734, 2017.

11. Kim Y, Choi JW, Lee JH and Kim YS: Expression of lactate $/ \mathrm{H}^{+}$symporters MCT1 and MCT4 and their chaperone CD147 predicts tumor progression in clear cell renal cell carcinoma: Immunohistochemical and The Cancer Genome Atlas data analyses. Hum Pathol 46: 104-112, 2015.

12. Choi JW, Kim Y, Lee JH and Kim YS: Prognostic significance of lactate/proton symporters MCT1, MCT4, and their chaperone CD147 expressions in urothelial carcinoma of the bladder Urology 84: 245.e9-245.e15, 2014.

13. Gerlinger M, Santos CR, Spencer-Dene B, Martinez P, Endesfelder D, Burrell RA, Vetter M, Jiang M, Saunders RE, Kelly G, et al: Genome-wide RNA interference analysis of renal carcinoma survival regulators identifies MCT4 as a Warburg effect metabolic target. J Pathol 227: 146-156, 2012.

14. Marchiq I and Pouysségur J: Hypoxia, cancer metabolism and the therapeutic benefit of targeting lactate/ $\mathrm{H}^{+}$symporters. $\mathrm{J}$ Mol Med (Berl) 94: 155-171, 2016.
15. Polański R, Hodgkinson CL, Fusi A, Nonaka D, Priest L, Kelly P, Trapani F, Bishop PW, White A, Critchlow SE, et al: Activity of the monocarboxylate transporter 1 inhibitor AZD3965 in small cell lung cancer. Clin Cancer Res 20: 926-937, 2014.

16. Guan X, Bryniarski MA and Morris ME: In vitro and in vivo efficacy of the monocarboxylate transporter 1 inhibitor AR-C155858 in the murine 4T1 breast cancer tumor model. AAPS J 21: 3, 2018.

17. Izumi $H$, Takahashi M, Uramoto $H$, Nakayama $Y$, Oyama $T$, Wang KY, Sasaguri Y,Nishizawa S and Kohno K: Monocarboxylate transporters 1 and 4 are involved in the invasion activity of human lung cancer cells. Cancer Sci 102: 1007-1013, 2011.

18. Lee JY, Lee I, Chang WJ, Ahn SM, Lim SH, Kim HS, Yoo KH, Jung KS, Song HN, Cho JH, et al: MCT4 as a potential therapeutic target for metastatic gastric cancer with peritoneal carcinomatosis. Oncotarget 7: 43492-43503, 2016.

19. Morais-Santos F, Granja S, Miranda-Gonçalves V, Moreira AH, Queirós S, Vilaça JL, Schmitt FC, Longatto-Filho A, Paredes J, Baltazar F, et al: Targeting lactate transport suppresses in vivo breast tumour growth. Oncotarget 6: 19177-19189, 2015

20. Zhang P, Ma J, Gao J, Liu F, Sun X, Fang F, Zhao S and Liu H: Downregulation of monocarboxylate transporter 1 inhibits the invasion and migration through suppression of the PI3K/Akt signaling pathway in human nasopharyngeal carcinoma cells. J Bioenerg Biomembr 50: 271-281, 2018.

21. Kong SC, Nøhr-Nielsen A, Zeeberg K, Reshkin SJ, Hoffmann EK, Novak I and Pedersen SF: Monocarboxylate transporters MCT1 and MCT4 regulate migration and invasion of pancreatic ductal adenocarcinoma cells. Pancreas 45: 1036-1047, 2016.

22. Rybakovsky E, Valenzano MC, DiGuilio KM, Buleza NB, Moskalenko DV, Harty RN and Mullin JM: Improving transient transfection efficiency in a differentiated, polar epithelial cell layer. J Biomol Tech 30: 19-24, 2019.

23. Rao DD, Vorhies JS, Senzer N and Nemunaitis J: siRNA vs. shRNA: Similarities and differences. Adv Drug Deliv Rev 61: 746-759, 2009.

24. Romøren K, Thu BJ, Bols NC and Evensen Ø: Transfection efficiency and cytotoxicity of cationic liposomes in salmonid cell lines of hepatocyte and macrophage origin. Biochim Biophys Acta 1663: 127-134, 2004

25. Yeung KT and Yang J: Epithelial-mesenchymal transition in tumor metastasis. Mol Oncol 11: 28-39, 2017.

26. Winer A, Adams S and Mignatti P: Matrix metalloproteinase inhibitors in cancer therapy: Turning past failures into future successes. Mol Cancer Ther 17: 1147-1155, 2018.

27. Pachmayr E, Treese $C$ and Stein U: Underlying mechanisms for distant metastasis-molecular biology. Visc Med 33: 11-20, 2017.

28. Meirson T and Gil-Henn H: Targeting invadopodia for blocking breast cancer metastasis. Drug Resist Updat 39: 1-17, 2018.

29. Sasaki S, Kobayashi M, Futagi Y, Ogura J, Yamaguchi H, Takahashi N and Iseki K: Crucial residue involved in L-lactate recognition by human monocarboxylate transporter 4 (hMCT4). PLoS One 8: e67690, 2013.

30. Tsai PC, Hsieh CY, Chiu CC, Wang CK, Chang LS and Lin SR: Cardiotoxin III suppresses MDA-MB-231 cell metastasis through the inhibition of EGF/EGFR-mediated signaling pathway. Toxicon 60: 734-743, 2012.

31. Shojaei F, Simmons BH, Lee JH, Lappin PB and Christensen JG: $\mathrm{HGF} / \mathrm{c}-$ Met pathway is one of the mediators of sunitinib-induced tumor cell type-dependent metastasis. Cancer Lett 320: 48-55, 2012.

32. Navarini NF, Araújo VC, Brown AL, Passador-Santos F, Souza IF, Napimoga MH, Araújo NS and Martinez EF: The EGF signaling pathway influences cell migration and the secretion of metalloproteinases by myoepithelial cells in pleomorphic adenoma. Tumour Biol 36: 205-211, 2015.

33. Levenson AS, Thurn KE, Simons LA, Veliceasa D, Jarrett J, Osipo C, Jordan VC, Volpert OV, Satcher RL Jr and Gartenhaus RB: MCT-1 oncogene contributes to increased in vivo tumorigenicity of MCF7 cells by promotion of angiogenesis and inhibition of apoptosis. Cancer Res 65: 10651-10656, 2005.

34. Payen VL, Hsu MY, Rädecke KS, Wyart E, Vazeille T, Bouzin C, Porporato PE and Sonveaux P: Monocarboxylate transporter MCT1 promotes tumor metastasis independently of its activity as a lactate transporter. Cancer Res 77: 5591-5601, 2017.

35. Majumder A, Ray S and Banerji A: Epidermal growth factor receptor-mediated regulation of matrix metalloproteinase- 2 and matrix metalloproteinase-9 in MCF-7 breast cancer cells. Mol Cell Biochem 452: 111-121, 2019.

This work is licensed under a Creative Commons Attribution-NonCommercial-NoDerivatives 4.0 International (CC BY-NC-ND 4.0) License. 